\title{
Manufacturing as a System-Determined Science
}

\author{
JOHN L. CASTI
}

\begin{abstract}
This paper addresses the general issue of manufacturing as a system-determined science. To adequately assess the degree to which systems thinking enters into considerations of modern manufacturing, several interconnected schemes are developed which, taken together, provide a taxonomy of manufacturing problems. It is shown that each manufacturing problem exists at a certain hierarchical level, the lowest being Raw Materials, the highest Values. Further, each problem can be labeled as a Design, Production, or Distribution problem, provided these terms are taken in a general sense. Finally, it is shown that each problem has associated with it one or more foundational system concepts (flexibility, complexity, adaptation, etc.), lending the problem its characteristic system flavor. Putting the hierarchical, Design-Production-Distribution and system concepts labels together supplies the basis for a classification scheme which, at the same time, enables us to unequivocally answer the question as to whether there is a significant systems component to most problems of modern manufacturing. There is!
\end{abstract}

\section{Manufacturing in Transition}

To the average Inlan on the street, mention of the word "Inanufacturing" likely conjures up visions of red-hot blast furnaces, clanking assembly lines, unfathomable machinery, and hosts of blue-shirted workers scurrying to and fro conveying components from one part of a dark, dingy, dirty factory to another. This image, fostered by the automobile manufacturing process in the early 1900s, is finally undergoing a long overdue refurbishing as a result of the introduction of widespread, cheap information processing and communication capacity into the industrial workplace. While it is true that manufacturing still consists of the eternal triangle, Design, Production, and Marketing, welded together by Planning and Management, the manner in which the manufacturing tasks are being carried out is undergoing a radical transformation, a transformation comparable in scope, perhaps, only to that experienced by agriculture a few decades ago. In short, the introduction of modern information processing facilities into virtually every nook and cranny of the manufacturing enterprise is resulting in a vision of the "factory-of-thefuture" as different from the "Model-T" image described above as a Model- $T$ is from a new Ferrari. We are moving into an era in which a very small fraction of the labor force will he involved in producing all the material goods that society can consume, the same situation we already have in agriculture. This paper is devoted to an exploration of the

Address reprint requests to John L. Casti, International Institute for Applied Systems Analysis, 2361 Laxenburg, Austria. 
"system-determined" characteristics of these future manufacturing enterprises and to pointing out the system problems that currently stand in the way of a realization of this vision.

How, then, has the introduction of advanced data processing capability changed the overall manufacturing process and what are the likely consequences for the shape of industrial production in the future? First let us take a look at some of the new or enhanced capabilities of tomorrow's factory:

- Flexibility - the possibility for producing products from an almost limitless variety of designs and materials: "economies of scope" replace economies of scale;

- Adaptability - ability to respond quickly to changing market demands and unexpected environmental fluctuations;

- Reliability - the capacity to more effectively schedule maintenance, increased use of automation (robots), and numerically controlled equipment all contribute to higher levels of reliability in finished goods;

- Efficiency - better use of machines, reduced inventories, and fewer stoppages for missing materials and parts are all results of the improved management capabilities emerging out of advanced software;

- Controllability-enhanced sensor technology, coupled with improved information processing, gives heretofore unprecedented management information and control over all operations.

In short, the factory of the future will be flexible and efficient, together with greater complexity of both product and process. Finally, such a factory will consistently produce high-quality products. Only the computer-integrated manufacturing system, with its vastly improved information and decision capabilities, offers a means to transform the factory of yesterday into this type of factory of tomorrow. But the shift in emphasis from product to process brings with it a shift from hardware to software, the ultimate consequence being an emergence of the crucial role of systems thinking in building the factory of tomorrow. In the final analysis, this means that manufacturing must become more of a science and less governed by "rules of thumb" and intuitive judgement. After all the smoke clears away, what remains is the need to develop a systems-based "theory of manufacturing". In what follows, we shall attempt to provide an "alphabet" from which such a theory may be composed.

\section{Factories of Tomorrow-A Skeletal Outline}

Prior to entering into a detailed consideration of manufacturing and manufacturing problems, it is useful to gain a bit of perspective by taking a look at what a typical manufacturing operation might look like 10-15 years from now. As we shall see in the next section, the overall issue of Manufacturing encompasses several levels ranging from raw materials considerations to social values, but the level of greatest concern and the level most of us intuitively feel represents "real" manufacturing is the level of the individual manufacturing unit, i.e., a plant or a firm. The skeletal outline presented here of how the plant of tomorrow will function touches only the mountain tops and gives a compact overview of the principal features distinguishing such a plant from its contemporary counterpart. For a detailed treatment along the same lines for a prototypical metalworking plant, we highly recommend the very extensive and perceptive report by Solberg et al. [1]. The manufacturing literature contains a variety of speculations and prognostications of similar nature; some of the best are found in $[2-4]$. 
The most distinguishing aspect of the manufacturing plant of the future is its heterarchic structure. The ability to transfer information almost instantaneously from one part of the process to another means that the traditional hierarchical, tree-structure, sequential manufacturing system will be transformed into a heterarchical, distributed, parallel-processing system capable of a high level of flexibility in producing a myriad of products with high quality and efficiency. The effective coordination of such a distributed process would be unthinkable, of course, without the information processing resources that have only recently become available. It is probably not an exaggeration to say that the ultimate aim of any decent theoretical study of such manufacturing processes is to devise a framework that enables us to understand how to configure the various components of the manufacturing process (design, production, distribution, management) to most efficiently and effectively employ the computing resources available. We shall have considerably more to say about this point later on, but for the moment let us consider a typical scenario for such a plant.

The process of designing a product will begin with an iterative dialogue between the designer and the computer (CAD). The designer will supply the product, concepts, and specifications, while the computer carries out design calculations and provides standardized information. During this process, the computer can be continually taking into account information on the manufacturing costs and capabilities needed to actually produce the product under design. The computer will then employ this information to generate a design that not only meets the product specifications, but also can be manufactured in some "optimal" way. It is important to note that this design phase of the process may be physically far removed from the actual plant facilities involved in the production of the product. Nevertheless, current information technology will enable the design computer to be in continual contact with the status of the plant and to employ this information as part of the design process.

At almost the same time the design process is going on, the production planning part of the system will use the design information to set up an optimized production plan to produce the product. This plan will involve selecting the proper equipment and processes, configuring the sequence of operations, choosing the operating conditions, etc. All of the design and production information will then be used to control the automatic machines that will actually perform the physical operations. Each of these machines continually feeds information about its status back to the production control system, which then performs dynamic adjustments to the production plan as needed.

While the production process is under way, the various machines will be carrying on self-diagnosis of their condition, and, if a failure is impending, they will perform automatic corrective actions. In addition, the machines will also carry out automatic quality control inspections at each stage of the product's manufacture, so that the final finished product will be fully inspected and conform to the original design requirements.

During the course of production, the distribution planning component of the systcm will be in communication with the production part, gathering information as to how to best allocate the finished product among various distribution centers. The distribution program must optimally balance current demands and order backlogs with available transportation facilities and costs to decide the optimal means for distributing the finished product among various distributors/consumers and inventory warehouses.

This brief skeletal outline of the operation of the factory of the future is notable for its reliance upon a high degree of communication, both within each major component and, more importantly, between components. Each stage must be planning its action upon 
information as to what's happening in the preceding stages with the loop being closed by management strategy reading the results of the distribution network (sales, protits, and the marketplace) and feeding this information back to the design stage.

Even in as sketchy an outline of tomorrow's factory as that given here, several dominant themes already emerge characterizing major departures of future factories' ways of doing business from the factories of today. Among the most significant features, we find the following:

- Speed-in today's factory, the typical throughput time from order placement to fulfillment is measured in weeks and months; in the future this time will be measured in hours and days. It is important to note here that the push for speed is only partly motivated by a desire to give better customer service. An equally important motivation is the need for better control and cost reduction (Just-intime inventory control, for example). The downside in this push for speed will be the emergence of serious bottlenecks in the process as operations that are now robust due to long leadtimes lose their stable character and become potential problems.

- Flexibility-many variations in product specifications that we now regard as exceptional will become normal in the future. As a result, equipment will be versatile enough that its cost can be amortized over many different products. In addition, to make small batch sizes economical, it will be necessary to reduce setup costs to the point where they are no longer a factor in the product cost calculations.

- Artificial (machine) intelligence-the factory operation described above relies heavily upon the ability to manage enormous quantities of data, and the capacity to transform that data into information and then into knowledge. Such a factory must ultimately reduce its dependence upon human judgment and interpretation, replacing it with a rational foundation for design, production, and distribution based upon process models and physical laws. To carry out this transformation from today to tomorrow will require a genuine science of manufacturing, not just a large body of experience.

- Integration of technologies-it is a commonplace today that factories are often unable to benefit from known technologies because these technologies do not comfortably fit together. Advances in highly specialized areas of research are wasted because bottlenecks at the interface impede effective utilization of the new technology. In the future factory, considerable attention will be given to integration of individual technologies into a harmonious whole. This will be a "rebuild-fromcommon-foundations" kind of interpretation, rather than a "paste together" sort $[1]$.

With the foregoing image of tomorrow's manufacturing enterprise in mind. let us now turn to a more detailed consideration of the types of systems problems such an organization will generate.

\section{A Taxonomy for Manufacturing Problems}

When we use the term "systems problem" to describe a manufacturing situation what do we really mean? Are there some identifying "fingerprints" that enable us to characterize certain aspects of manufacturing as "systems" aspects, while denying this label to other problems? Basically, is there such a thing as a "system" theory of manufacturing distin- 
guishable from any other garden-variety theory of manufacturing? Our contention is that the answer to all these queries is yes, but it is an answer that comes in several parts.

Before embarking upon a justification of our claim, let us clear the air a bit regarding what we think of as a "systems" problem. Basically, we consider a systems problem to be one in which the emphasis is placed upon function rather than form; process rather than structure; software rather than hardware. Of course, this is a crude, vague sort of classification, but is evocative of the features characterizing a system-dominated problem. We shall elaborate and embroider upon these basic ideas within the context of manufacturing as we proceed, each level of elaboration further refining the systems nature of manufacturing operations.

At the outset, we must recognize that the very concept of a manufacturing system is a multifaceted one and that the identification of systems problems is very much dependent upon our perspective of the overall process of manufacturing. We have found it useful to consider manufacturing as an activity to be stratified into the layers depicted in Figure 1. Here we have indicated that the systems-determined aspects of manufacturing tend to emerge at the middle levels of the diagram, while the upper and lower levels are dominated by problems in which the systems aspect is of lesser importance. Notice that we say "of lesser importance" and not "unimportant." What determines a systems problem in manufacturing is the relative emphasis upon issues of process and function, grounded in constraints from the natural sciences, requiring a knowledge of several disciplines for their treatment. The dominant problems at the lower levels are primarily disciplinary (e.g., physics, chemistry, materials science) in orientation, focusing upon physical properties and structures; upper level problems, for the most part, emphasize the social sciences and humanities (e.g., philosophy, economics, theology) and are really transdisciplinary in nature, stressing neither process nor structure. Thus, just as in chess where the action is in the middle game, so it is in manufacturing: the systems problems reside in the

$\underbrace{\text { (macro) }}_{\text {(meso) }} \begin{array}{llll}\text { IX } & \text { Values } & \begin{array}{l}\text { socio-cultural } \\ \text { impacts }\end{array} \\ \text { VIII } & \text { World Industry } & \text { global economy } \\ \text { VII } & \text { Manufactured foods } & \text { national economy } \\ \text { VI } & \text { Industry } & \text { national industry } \\ \text { V } & \text { Local Industry } & \text { regional industry } \\ \text { IV } & \text { Finished Product } & \text { firm } \\ & & & \\ \text { III } & \text { Components } & \text { assembly line } \\ \text { II } & \text { Parts } & \text { Materials } & \text { shop floor } \\ \text { I } & & \text { raw materials }\end{array}$

(social science, transdisciplinary)

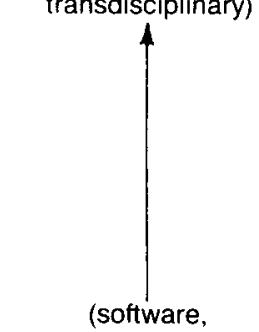

natural science, multidisciplinary, process, information)

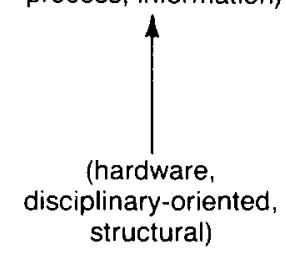

Fig. 1. Manufacturing system stratification. 
midrange of our hierarchy, roughly speaking levels II-V, and especially in the paths linking these levels. As indicated in the diagram, there are feedbacks and feedforwards from all levels, which no decent overall theory of manufacturing can afford to neglect. Nevertheless, we feel that the real power of system thinking can most effectively be employed in the problems arising at the midlevels, and we shall focus most of our remaining remarks there.

To further sharpen and refine our thinking about the systems nature of things in manufacturing, it is helpful to recognize that each layer of the stratification of Figure 1 can be subdivided into three components which we can abstractly label "Design" (D). "Production" (P), and "Marketing"/"Distribution" (M). At the level of the firm itself (level IV), these labels have their everyday interpretation; at other levels we shall have to intcrpret them in a fashion consistent with that level. For example, at the national economy level (level VII), we have

"Design" $\leftrightarrow \begin{aligned} & \text { planned structure and operations of the economy as envisioned by } \\ & \text { government policymakers, }\end{aligned}$
"Production" $\leftrightarrow \quad \begin{aligned} & \text { actual operation of the economy, i.e., actual mechanisms } \\ & \text { employed in the production of goods and services, }\end{aligned}$
"Marketing" $\leftrightarrow$ distribution of goods and services to consumers, together with the
feedback from consumers to decisionmakers and producers.

As another example consider raw materials (level I). Here we have

"Design" $\leftrightarrow \begin{aligned} & \text { specifications and/or determination of properties of a given } \\ & \text { material, }\end{aligned}$
"Production" $\leftrightarrow$ means for actual construction or extraction of the material,
"Marketing" $\leftrightarrow$ means of conveying materials to users (manufacturers)

We leave it to the reader to fill-in appropriate $\mathbf{D}-\mathbf{P}-\mathbf{M}$ interpretations for other levels in our stratification. The important point is that such interpretations can be given and they enable us to see more clearly the systems aspects of problems at a given level.

Neither the "I-IX" nor the "D-P-M" subdivision of manufacturing problems makes any distinction between those problems which we would term "systems-determined" and those that are not. To get at the systems aspect of things, we introduce a third subdivision aimed at isolating those conceptual features of manufacturing problems that give the problems a distinctly systems flavor. It is possible to identify at least eight conceptual issues whose emphasis in a given manufacturing problem stamps it as primarily a "systemsdetermined" problem. These concepts are

- Efficiency/Optimality (EFF);

- Flexibility/Adaptability (FLX);

- Complexity (COM);

- Vulnerability/Resilience (VUL); 
- Reliability (REL);

- Uncertainty/Fuzziness (UNC);

- Self-Organization/Replication (SLF);

- Performance (PRF).

Let us examine the underlying content of each of these themes in the context of manufacturing.

A. Efficiency/Optimality - traditionally when one thought of a systems problem in the manufacturing areas, the idea that sprung to mind was the effective utilization of some resource: manpower, money, time, and/or materials. Problems of this kind include optimal scheduling of machines in a job-shop, optimal levels of inventory, maximal use of raw materials in a stock-cutting operation, minimal transportation costs in warehousing finished goods, and so forth. In all cases, the problem emphasis is upon optimizing a given quantity subject to resource, material, space, and time constraints of various sorts. The traditional tools of operations research such as linear programming, network flow analysis, PERT, and dynamic programming were originally developed to handle systems problems focused upon the concept of efficiency.

B. Flexibility/Adaptability-probably the most overworked word in the manufacturing lexicon today is "flexibility," used to convey a whole host of ideas centering upon the theme of an integrated, computer-controlled complex - automated material handling devices and machine tools that can simultaneously produce medium-sized volumes of a variety of parts types. In the manufacturing environment, we can identify at least eight types of flexibility [5]:

i. Machine flexibility characterizing the ease of making changes required to produce a given set of parts types.

ii. Process flexibility involving the ability to produce a given set of parts types, each possibly using different materials, in several ways.

iii. Product flexibility measuring the ability to changeover to a new set of products very economically and quickly.

iv. Routing flexibility dealing with the ability to handle breakdowns and to still produce a given set of parts types.

v. Volume flexibility indicating the capacity to profitably operate a manufacturing systems at different production volume.

vi. Expansion flexibility expressing the capability of building a system and expanding it, as needed, easily and modularly.

vii. Operation flexibility associated with the ability to interchange the ordering of several operations for each part type.

viii. Production flexibility, a measure of the universe of parts types that the manufacturing system can produce.

Of course, not all of these notions of flexibility are independent. Figure 2 displays the relationships among the various types of flexibility, where the arrows denote "is necessary for."

Systems problems emphasizing flexibility focus upon means to enhance one or more of the above flexibility capacities, while at the same time not degrading any of the others. For example, keeping routing options open and not predetermining either the "next" operation or the "next" machine increases operation flexibility and, as a result, improves overall production flexibility, too. One of the major difficulties with flexibility-dominant 


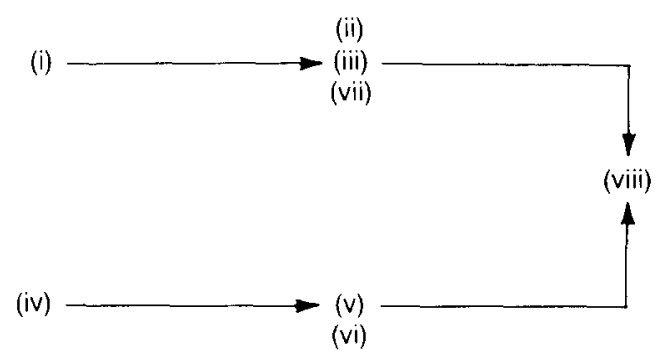

Fig. 2. Dependencies among notions of flexibility

problems is the lack of a consistent measure of flexibility that would enable one to transfer back and forth between the various types, i.e., a "common currency" of flexibility, so to speak.

C. Complexity-complexity is a term almost as overworked as flexibility, characterizing some measure of the difficulty in understanding the products and processes of manufacturing operations. It has been argued elsewhere [6] that, in general, complexity is a contingent property of a system, emerging from that system's interaction with another. In the predominantly engineering environments of manufacturing operations, this contingent property is mostly hidden, and it makes sense to think of complexity as an intrinsic system property characterized by some combination of the number of system components, the nature of their interconnection, the dynamical flow of information between the parts, and the interaction between the various hierarchical levels comprising the system.

In the manufacturing setting, complexity emerges because the traditional processes involving simple operations such as machining, forming, joining, and the like have been augmented or replaced by other novel techniques such as lasers for cutting, plasma etching techniques, and ion beam pattern processing. In addition, new materials like plastics, ceramics and carbon fibers interact with the manufacturing process in many unexpected ways contributing to the overall complexity of the manufacturing.

Generally speaking, complexity-dominated manufacturing problems emphasize questions pertaining to either the reduction in production and/or process complexity by, for example, introduction of automated processes, combinations of processing operations, alternate scheduling procedures, and the like or to the effect on system complexity of changes directed to other properties like flexibility, resilience, and/or optimality. As an illustration, it seems to be a folk theorem that system complexity and system stability are directly related, i.e., increased complexity generates increased stability. The intuitive argument given to support this contention is that greater complexity generates a denser network of connections between system components, and this higher level of connectivity results in the system being more capable of absorbing potentially destabilizing disturbances. Unfortunately, without a clearer concept of the notion of complexity, its relationship to the interconnection of the system components, and the impact of the connective structure upon the type of stability being considered, it is impossible to either confirm or deny the claimed complexity/stability relationship. An account of this problem in the context of ecological networks and food webs is given in [7], but the corresponding concepts and results for manufacturing systems have yet to be developed.

$D$. Vulnerability/Resilience---what is the degree to which a system can sustain disturbances and disruptions in its normal operating environment and continue to perform its designated function? 'This is the essence of what we mean by a system's vulnerability, while the capacity of the system to absorb perturbations and continue functioning is a 
measure of its resilience. Both of these notions are, of course, particular cases of the general problem of system stability, i.e., if we change something about the system, when does it matter?

In the manufacturing context, issues of vulnerability are among the most frequent and important that the system manager faces. Equipment breakdowns, delays in supplies, unexpected design changes, market fluctuations, variations in raw material quality, and a thousand other minor and major shocks are continually impacting the system, and it is of the utmost importance that the overall manufacturing operation be structured in a manner that makes it relatively cheap and easy to accommodate such disturbances. The heart of the vulnerability question in manufacturing lies in the determination of good ways to configure the system to provide it with a sufficiently large safety margin to enable it to "roll with the punches" continually impacting it from an uncooperative external environment.

From what has been said earlier, it should be clear that the notion of system vulnerability is closely intertwined with ideas of flexibility, adaptability, and complexity; in fact, it seems difficult to imagine how to attack one of these problem areas without consideration of the others. But, as with flexibility and complexity, so it is with vulnerability and resilience, and we have, as yet, no coherent measures of a manufacturing system's vulnerability, nor any concrete idea of the nature of the relationships linking these system concepts.

E. Reliability - in manufacturing, reliability refers to the system's ability to maintain uniform quality and consistent delivery schedules in the face of changing demands and/or operating circumstances. In the past, the assembly line and numerically controlled machine tools generated major advances in quality because of product standardization; the future challenge will be to achieve even higher levels of reliability in quality without standardization or long learning curves, while working to finer tolerances and scales. One major innovation in this direction is the widespread introduction of robots into the manufacturing process; another is the introduction of new analytical design tools that will automatically prevent the designer from making inadvertent errors, or from specifying tolerances that are either too loose or too tight.

Again, it is clear that reliability is closely related to other system concepts, especially vulnerability and resilience. But the flavor here is somewhat different, as reliability focuses upon consistency in the product and its production process, while the earlier concepts emphasized the viability of the overall manufacturing operation. But it is really more a question of degree rather than kind, and we can readily expect advances in understanding system reliability to provide insight into vulnerability, and conversely.

F. Uncertainty-many of the types of disturbances we have referred to in connection with the concepts of vulnerability, resilience and reliability have their origin in stochastic phenomena affecting the manufacturing system. Random machinery failures, unpredictable market demands, fluctuating supplier schedules, and the like are well-known phenomena in manufacturing circles, and many of the classical OR tools have been developed to address them. But there is another kind of uncertainty concept, not having its source in any stochastic features, but rather in a certain linguistic "fuzziness" in the very problem description itself. In such situations, the variables describing the problem are themselves only vaguely defined, and the task is to create a type of calculus for combining and operating with such "fuzzy" variables.

As an illustration, consider the concept of a "tall" man. It's clear that every man has a well-defined, definite height measured on some scale, say so many centimeters. What is not clear is the notion "tall." Is it all men over $190 \mathrm{~cm}$, over $188 \mathrm{~cm}$, and less 
than $200 \mathrm{~cm}$, or what? It's impossible to say, and all we can do is assign a degree-ofmembership function to the set consisting of tall men. This is the essential idea underlying the theory of fuzzy sets, as developed in recent years by Zadeh, Negoita, and others.

Many problems in manufacturing have this second kind of uncertainty as an essential part of their specification. We want to "rapidly" produce products of "good" quality in "small" batches at a "low" cost. This is a typical description of a manufacturing operation given to the media by a CEO and, as can be clearly seen, is riddled with vaguely defined linguistic variables. Many of the obstacles standing in the way of a truly flexible automated factory hinge upon the development of good software capable of dealing with this type of uncertainty. The fuzzy set concepts are one direction, the "possibility" theory of Klir is another, and there may be many more. What is needed for the specific problems of manufacturing remains, at this date, an open question.

$G$. Self-Organization/Replication-two of the characterizing features of living organisms are their abilities to repair structural damages and to reproduce themselves. When one reads descriptions of envisioned future factories $[6,8]$, a striking aspect of these projective speculations is how similar they sound to a description of a living organism. While the matter of "self-reproducing factories" sounds somewhat fanciful, there is ample reason to take seriously the idea of factories that engage in automated sensing of their operating environment and acts of self-repair and self-reconfiguration (organization) according to the ambient conditions and circumstances.

An essential aspect of the concept of self-organization is that, at some level of systems complexity (however it is measured), new forms of organization and functions emerge, forms whose very existence is determined by the inability of the system's components to adequately adapt at a lower level of organization. With the added complexity inherent in the advanced information processing, communication, and automation available, issues of self-organization and its attendant concepts of bifurcation, adaptation, and self-repair will play an increasingly important role in future manufacturing system studies.

$H$. Performance - one of the thorniest aspects of many system problems, especially those involving social and behavioral phenomena, is the determination of a yardstick by which to measure the "goodness" or "badness" of the system's behavior; this is no less true in manufacturing. How to evaluate prospective changes such as introduction of robots, flexible machinery equipment, new process techniques, exotic materials, JIT inventory procedures, and so on is likely the most difficult task facing the manager of a manufacturing operation. It is probably true that the further down the hierarchy of Figure 1 you go, the casicr it is to identify a precisc evaluation criterion, s.sil as number of items produced per unit time, unit profits, production efficiency, etc., but even at the lowest levels the problem is by no means totally straightforward. And at higher levels where one must balance the manufacturing system's needs with the role the system plays in the global operating environment, it is far from clear what measure adequately reflects the system's overall "performance." What is clear is that much more than just quality, productivity, and profitability are involved.

So, the final jewel in our crown of system concepts in manufacturing, and the concept to which all others are subservient, is the notion of a criterion (or criteria) of performance. The development of such criteria is the uninvited guest at the banquet table in the analysis of any problem involving the concepts and levels introduced above.

\section{System Problems in Manufacturing: A Generative Mechanism}

The three levels discussed in Section 3 comprise a taxonomy for manufacturing problems and provide us with an algorithmic, or generative, procedure for formulating an almost infinite variety of systems-determined manufacturing issues. We first summarize 
the levels of taxonomy by the chart in Table 1 . Our almost automatic mechanism for generating manufacturing problems with a systems flair follows a procedure that is the essence of simplicity: pick one item from each of the columns of Table 1 and combine them to formulate the problem. The procedure can be used in two directions: as just indicated, to generate new system problems in manufacturing or, in the opposite direction, to classify or categorize an existing problem.

As illustration of both uses of the "taxonomy principle," consider the following:

\section{GENERATIVE MODE}

1. (IX/D/COM - -this would be a problem involving societal values (level IX), their determination ("Design"), and the complexity (COM) in the context of manufacturing systems. A typical problem of this sort would involve the way in which manufacturing systems impact the overall goals of society, how to (re-)design those goals to take into account the anticipated role of manufacturing in the future, and the complexity associated with the interaction between a technological object (the manufacturing system) and a social organism (society, at large).

2. (IV/P/FLX) - this code represents a problem involving production $(\mathrm{P})$ flexibility (FL) in an individual firm (level IV). A prototypical problem of this sort is to determine the increase in overall productive capacity by introduction of automated machining operations, emphasizing the enhanced flexibility of the plant.

3. (I/M/REL - - in this case, the code suggests a problem dealing with the reliability (REL) of the distribution network (M) of raw materials (level I) needed for manufacturing operations. This type of problem is of central importance, for instance, in consideration of implementing a JIT inventory control system.

Neglecting codes involving interfaces and/or multiple column entries, the above scheme admits 216 separate classification codes for systems problems in manufacturing, with each of these cases capable itself of supporting a vast array of individual problems. However, as noted earlier, it is also possible to run the foregoing coding scheme in reverse to classify a given manufacturing problem. Let us consider a few examples.

\section{CLASSIFICATION MODE}

Example 1. In discussion with the management of a large Japanese electronics firm, the plant manager remarked that one of his biggest problems is how to increase engineering productivity. By this he meant the speed and efficiency by which the engineers could modify circuit designs and production procedures to suit the specific needs of individual customers. Such a problem is most suitably addressed at the level of the individual plant

TABLE 1

Manufacturing System Taxonomy

\begin{tabular}{llll}
\hline Hierachical & \multicolumn{1}{c}{ Level } & \multicolumn{1}{c}{$\begin{array}{c}\text { Functional } \\
\text { Level }\end{array}$} & \multicolumn{1}{c}{$\begin{array}{c}\text { Conceptual } \\
\text { Level }\end{array}$} \\
\hline (IX) & Values & & Efficiency (EFF) \\
(VII) & World industry & & Flexibility (FLX) \\
(VII) & Manufactured goods & Design (D) & Complexity (COM) \\
(VI) & Industry & Production (P) & Vulnerability (VUL) \\
(V) & Local industry & Marketing (M) & Reliability (REL) \\
(IV) & Finished product & & Uncertainty (UNC) \\
(III) & Components & & Self-organization (SLF) \\
(II) & Parts & & Performance (PRF) \\
(I) & Materials &
\end{tabular}


(level IV), and involves both Design (D) and Production (P) aspects. Finally, the essential nature of the problem is the Efficiency (EFF) of the plant, although the problem also touches upon issues of flexibility (FLX) and overall factory performance (PRF). Consequently, it would be appropriate to assign this problem to the class (IV/D-P/EFF-FLXPRF), illustrating already the point noted earlier than the most system-determined of system problems are those involving the interfaces and/or combinations between the various levels and categories.

Example 2. A problem of a quite different character arose during conversations with the managing director of another large Asian electronics company. It was mentioned that the first priority of the firm, at the moment, was the installation of a computer information system to monitor the various stages of design, production, and distribution. Then came the surprisingly candid admission that once the hardware was in place, the firm would be faced with the difficulty of what to do with the data acquired. So, the essence of this problem is how to transform data into information. In terms of our taxonomic scheme, this is again a factory-level problem (level IV), involving all three functional levels (DP-M). The primary system concept at work here is the ideas of complexity (COM): the objective of the management information system is to reduce a collection of increasingly inter-dependent processes to manageable (read: simple) levels. Thus, the proper code for this problem is (IV/D-P-M/COM).

Example 3. At an American computer company in California, the manager of advanced manufacturing related the procedures followed in the construction of their very low-volume (500 units/year), high complexity machines. He stressed the point that "manufacturability"/"testability" was the current priority concern for their group. This question essentially deals with the design of the computer components in such a way that the physical structure facilitates easy construction and testing. Relating this problem to our taxonomic structure, it involves the computer Components (level III), and the choice of their Design (D) to reduce Complexity (COM) to facilitate their Reliability (REL). Putting these observations together, a suitable category for this problem is (III/D/COM-REL).

Examples of the above sort could be multiplied severalfold, but the basic principles are already clear. The question that remains is what to do with one of these myriad system problems once it has been identified and classified. This is a question not of labels or codes, but of tools, the final ingredient in our dissection of manufacturing from a systems perspective.

\section{System-Theoretic Tools, Techniques, and Structure in Manufacturing}

The preceding development has amply demonstrated the inherent systems nature of many manufacturing problems; but problems without solutions are like bread without butter. Consequently, in this section we explore the equation

$$
\text { problems }+ \text { tools }+ \text { a world view }=\text { insight, }
$$

emphasizing the spectrum of system-theoretic tools that can be brought to bear upon manufacturing problems of the sort considered above.

In the above equation, an indispensable role in determining the nature and degree of insight that can be gained about any problem is played by one's scientific Weltanschauung, or "world view." When translating a problem statement into a formal mathematical structure, the world view is represented by the type of formal mathematical system chosen to reflect the features of the problem. In turn, this mathematical world view then dictates the kinds of question that can be asked and the tools and techniques 
that can be used in seeking insights and answers. Schematically, we have the situation depicted in Figure 3.

A crucial aspect of the success or failure of any modeling venture is the choice of the "right" mathematical structure to employ on the right side of this diagram. When faced with a new class of problems like those arising in manufacturing, where a substantial body of past work is unavailable to draw upon, it may be necessary to employ an intermediate step as shown in Figure 4. Here the idea is to select a surrogate system $X$ whose mathematical representation is relatively well-understood (the coding/decoding $\beta$ ). If we can construct the "dictionary" $\alpha$ between $X$ and our manufacturing system of interest, then by composition we are able to implicitly generate the map $\gamma$ of primary interest. A research program based upon the exploitation of Figurc 4 examining various choices for the system $X$ (biological, computer, electrical circuit, language) is given in an earlier report [6]. The point to be emphasized is that there is no such thing as the "correct" or "right" way to tackle any of the problems arising in manufacturing; each world view, or "paradigm," generates its own slice or reality, and it is the task of the analyst to piece together enough such slices to create a picture of great enough detail for the task at hand. Now let us turn to an examination of some types of world views for manufacturing.

It seems to be convenient to divide the set of mathematical paradigms for manufacturing into four principal components. For sake of nomenclature, we term these classes Operations Research (OR), Computer Sciences (CS), Control Theory (CT), and System Theory (ST). We briefly indicate the principle types of problems and specific techniques characterizing these paradigms.

A. Operations Research-problems in this category basically revolve around issues of planning and scheduling. Thus, we have issues of manufacturing system planning involving the specification and organization of manufacturing resources needed to meet somelong-term production goals; production planning which takes facilities design as given, and sets aggregate production rates to be consistent with facilities capacity and demands; flow planning for determination of actual production batches in a manner consistent with both the production plan the resource constraints; and finally, scheduling involving the implementation of the flow plan and the sequencing and coordination of production activities.

Methodologically speaking, the techniques that are employed in the OR-based problems are the traditional OR tools: resource allocation, scheduling theory, inventory control, queuing theory, mathematical programming, decision analysis, and so forth. In the context of modern manufacturing, the OR problems are exactly the same problems as those encountered in the traditional manufacturing environments of the 1950s. The advent of $\mathrm{CAD} / \mathrm{CAM}$ and flexible manufacturing systems has not changed the nature of those problems one iota: a scheduling problem is still a scheduling problem and what was difficult in the 1950s is still difficult today. In fact, the problems may be even more

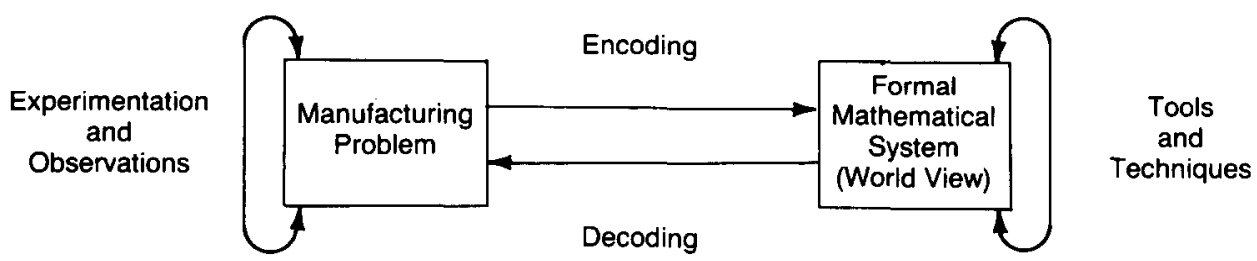

Fig. 3. The mathematical modeling relation for manufacturing. 


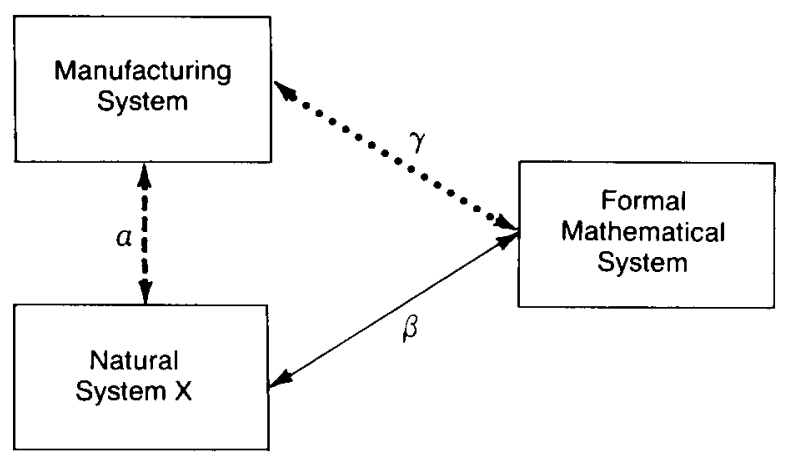

Fig. 4. Surrogate system model.

difficult today because of the higher levels of information processing and automation found in modern manufacturing environments.

As illustrations of some of the problems on the OR research agenda today, we have:

i. Aggregate production smoothing-development of means to dynamically smooth production in an uncertain environment over multiple production stages; development of means to aggregate products for production planning taking into account lot-size factors.

ii. Lot-sizing and reorder intervals - how to link lot sizing with aggregate production rates; determination of the relationship between lot sizes and machine sequencing.

iii. Planned lead times - how to set planned lead times for flow planning; how to integrate lead times with lot sizing, scheduling, and production planning; how to use lead times to set "promise dates" for customer deliveries.

iv. Protection stock-how to deal with production disruptions and delays; how to spread protection stock across many production steps; how to integrate protection stock with production planning and scheduling; determination of the relationship between protection stock and planned lead times.

v. Scheduling - how to integrate scheduling activity with planning; how to reschedule dynamically; how to create stable flow plans; how to schedule rework.

While the foregoing OR problems concentrate upon operational controls design, there are a corresponding set of issues for facilities, product, and process design.

$B$. Control Theory - during the 1960s, considerable advances were made in the methods of optimal control, stimulated mainly by problems in aerospace and mechanical engineering. Currently there is a movement underway to acquaint the controls community with manufacturing problems that possess the qualitative concepts associated with traditional control problems: complexity hierarchy, uncertainty, feedback. It is already clear that standard control theory techniques usually do not apply in a straightforward manner to manufacturing problems; this is not surprising since standard techniques have been developed for standard problems outside manufacturing. Thus, new standard techniques will have to be developed if control theory is to contribute in a major way to manufacturing.

Let us just briefly indicate some of the sorts of manufacturing questions of current interest to control engineers.

i. Machine control-calculation and implementation of optimal robot arm trajectories; control of cutting tools; control of furnaces and other steps in semiconductor fabrications. 
ii. Flexible manufacturing control-sequencing parts, fixture, and operations in a FMS; determination of optimal routes for transport carts; feedback monitoring of component quality in a FMS.

iii. Production control-development of factory-level models that integrate actual resource capacity with production requirements; determination of "just-in-time" material control in high uncertainty environments; decomposition of production lines into two-machine, one-buffer subsystems.

C. Computer Science-the main manufacturing problems of this type center upon the development of new computer languages and/or operating systems specifically designed for manufacturing and the use of $\mathrm{AI} /$ expert system techniques for manufacturing. As representative of the first type of problem, the U.S. Air Force is currently supporting a project called ICAM devoted to production of a language that is specifically aimed at making the integration of design and production easier. Similarly, due to the increasing level of decentralization in modern manufacturing systems, there is heightened interest in work on new operating systems to serve the heterarchic structure of many current manufacturing operations. Much of this work is aimed at dealing with the inherent information exchange problem emerging from anon-hierarchic decisionmaking environment.

Problems in the $\mathrm{AV} /$ expert system area involve the need to develop situation (not pattern) recognition programs, i.e., programs that recognize situations that threaten the continued viability of the manufacturing system. Other AI-oriented manufacturing problems focus upon the need for failure detection and correction programs. Here the work is devoted to means for automatic recognition of failures, as well as determination of remedial action and operating regimes under a total or partial failure mode.

D. System Theory-in at least partial contrast to the OR, Control Theory, and Computer Science-type manufacturing paradigms, a System Theory world view focuses more upon paradigm construction than upon techniques and algorithms associated with a given framework. Roughly speaking, in OR/CT/CS a particular formal structure is selected (linear program, set of differential equations, $M / M / 1$ queue or whatever) and the basic problems revolve about how various manufacturing questions can be addressed within the given paradigm (or a minor variation thereof). In short, one starts with a framework, or point of view, toward manufacturing processes and explores how the important concepts and issues of manufacturing fit into this framework.

The central issue in System Theory for manufacturing is quite different in spirit: start with all the manufacturing concepts and problems that matter and seek those paradigms within which these concepts can be consistently accommodated. The types of concepts that one starts with include notions such as complexity, flexibility, self-repair, adaptability, self-regulation, reliability, resilience, and performance. The objective is to develop a set of paradigms for manufacturing systems that will given an objective, precise, consistent, and useful meaning to these every-day terms. Since we are interested in making predictions about real manufacturing systems, what is sought are formal mathematical structures within which the concepts show up as relations between the elements comprising the mathematical formalism. The problem, at present, is that no one has any really good idea of what such a formal mathematical structure would look like for a modern manufacturing system. So, the only way to proceed is to argue by analogy with other types of natural systems that we do have such paradigms for. This is the essential content of Figure 4 and represents what amounts to a relational rather than structural, view of a system in the sense that we deal with classes of systems possessing some functional 


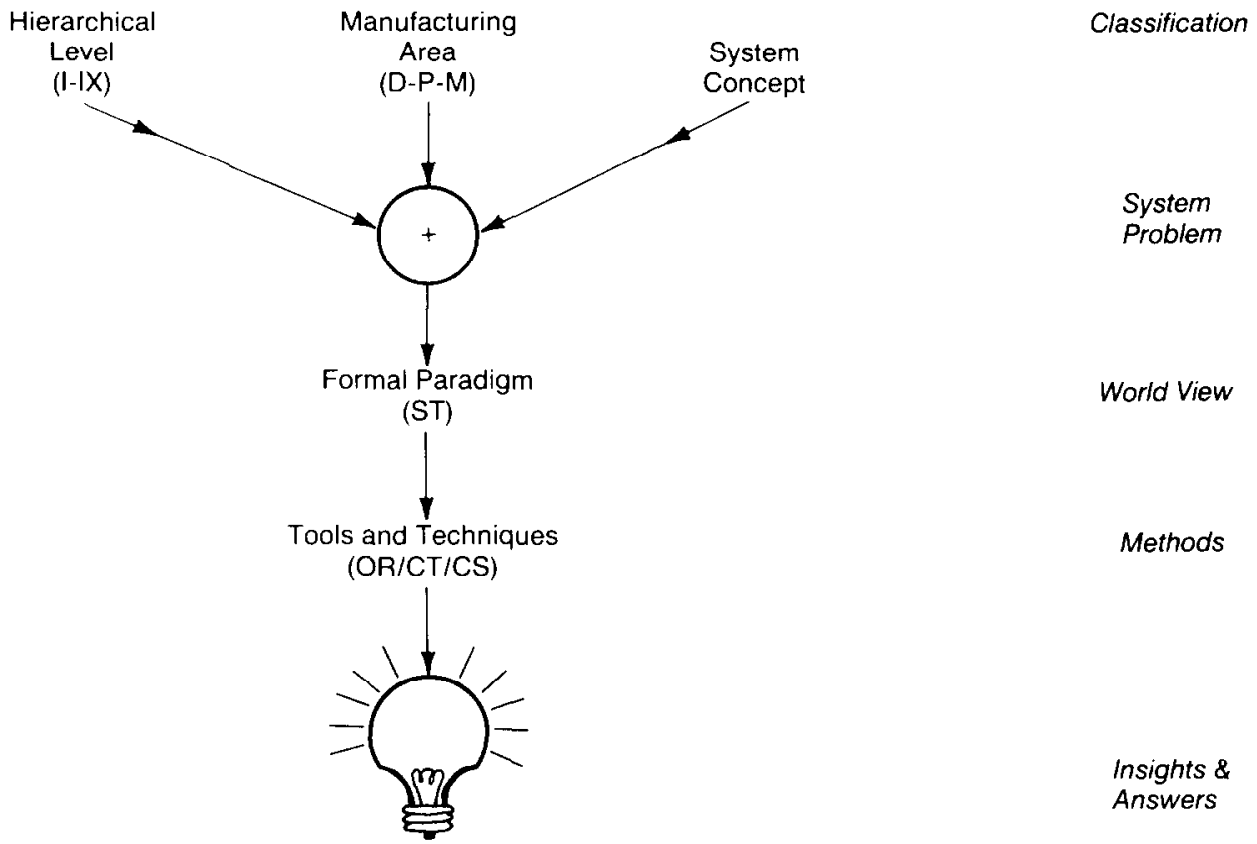

Fig. 5. A systems view of manufacturing.

similarities and neglect all aspects of physiochemical structure. A detailed account of this view is expressed in [9].

\section{Taxonomy and Technique: A Summary}

The foregoing sections have outlined a scheme for classification of systems-determined manufacturing problems, and have presented a rough breakdown of the tools and techniques for their resolution. At this point it is useful to compactly summarize the ideas and arguments in Figure 5. Here we see very explicitly that, as with the Holy Trinity, so with Manufacturing: to gain useful insights into manufacturing systems three ingredients are necessary: a system problem, a formal world view, and a method for exploring the implications of that view. Nothing less will suffice in any systems-based attack on the manufacturing issues of today.

We have now arrived at the point where we can confidently answer the main question of this study: manufacturing is indeed a systems-determined science, and there is no shortage of important unsolved problems for organization or individuals with a system orientation to tackle. Virtually everything remains to be done with the nuggets still lying on the surface waiting to be picked off by the first explorers of this uncharted and seemingly unlimited terrain.

\section{References}

1. Solberg, J., et al., Factories of the Future: Defining the Target, CIDMAC Report, Computer Integrated Design Manufacturing and Automation Center, Purdue University, Lafayette, IN, January 1985.

2. Bloch, E., Workplace of the Future, IEEE Transactions on Industry Applications IA-20, 8-10 (1984).

3. Suh, N. P., The Future of the Factory, Robotics and Computer-Integrated Manufacturing 1 (1984), 39-49.

4. Yoshikawa, H., Rathmill, K., and Hatvany, J., Computer-Aided Manufacturing: An International Comparison, National Academy Press, National Research Council, Washington, DC, 1982.

5. Browne, J., et al., Classification of Flexible Manufacturing Systems, in Manufacturing Systems: Context, Applications, and Techniques, V. Vignell, eds., Blackwell, Oxford, 1985. 
6. Casti, J., Metaphors for Manufacturing: What Could It be Like to be a Manufacturing System?", Technological Forecasting and Social Change 29, 241-270 (1986). Also IIASA WP-85-75, October 1985.

7. May, R. M., Stability and Complexity in Model Ecosystems, Princeton University Press, Princeton, NJ, 1973.

8. Hatvany, J., et al., The Computer-Controlled Manufacturing Cell- $-\Lambda$ chievements, Possibilities, and Perspective, Proceedings of 9th IFAC Congress, Budapest, July 1984.

9. Rosen, R., Fundamentals of Measurement and Representation of Natural Systems North-Holland, New York, 1978.

\section{Supplementary References}

While not directly cited in the text, the following publications have proven especially helpful in assessing the systems role of the future of manufacturing.

10. Merchant, E., Flexible Manufacturing Systems: Robotics and Computer Automation, Annals AAPSS 47, 123-135 (1983).

11. Morse. A.. A System for Embedding Data Displays in Graphical Contexts. Technical Report. 84-7-1. Visual Intelligence Corp., Amherst, MA, July 1984.

12. Hatvany, J., and Lettner, F., The Efficient Use of Deficient Knowledge, Annals CIRP 32, 423-425 (1983).

13. Goldhar, J., and Jelinek, M., Plan for Economies of Scope, Harvard Business Review. (Nov.-Dec.), 141-148 (1983).

14. Abraham, C., et al., Factory of the Future: A Research Agenda for Models to Plan and Schedule Manufacturing Systems, NSF Ad Hoc Committee Report, 4 March 1985.

15. Gerschwin, S., et al.. A Control Perspective on Recent Trends in Manufacturing Systems, MIT Laboratory for Information and Decision Systems Report LIDS-P-1408, August 1985.

16. Goldhar, J., and Burnham, D., Changing Concepts of the Manufacturing System, in US Leadership in Manufacturing, U.S., National Academy of Engineering, 18th Annual Meeting, 1984.

17. Gunn, T. G., The Mechanization of Design and Manufacturing, Scientific American 247, 87-108 (1982).

18. Chatterjee, A., et al., Manufacturing Flexibility: Models and Measurements, ORSA/TIMS Conference on Flexible Manufacturing Systems, University of Michigan, August 1984.

19. Whitney, C., Control Principles in Flexible Manufacturing, Journal of Manufacturing Systems 4, 157-166 (1985).

20. Gerschwin, S. Opportunities for Control in Manufacturing, IEEE Transactions on Automatic Control AC30, 933 (1985).

21. Gerschwin, S., Ramakrishna, A., and Choong, Y., Short-Term Production Scheduling of an Automated Manufacturing Facility, IBM Journal of Research and Development 29, 392-400 (1985).

22. CAM: An International Companion, Special Report 740, American Machinist (Nov.), (1981).

23. Kaplan, R., Measuring Manufacturing Performance: A New Challenge for Managerial Accounting Research, Accounting Review 58, 686-705 (1983).

24. Nishiyama, K., An Evolutionary Theoretical Model of Firms in an Industry: The Replicon Model, IEEE Transactions on Systems, Man and Cybernetics SMC-15, 662-665 (1985).

25. Bioch, E., Manufacturing Technologies, speech to Convocation of Engineering Academies, London, June 1985.

26. Yoshikawa, H., Flexible Manufacturing in Japan, Proceedings of the 9th IFAC Congress, Budapest, July 1984.

27. Yoshikawa, H., General Design Theory and a CAD System, in Man-Machine Communications in CAD/CAM, T. Sata and E. Warman, eds., North-Holland, Amsterdam, 1981.

28. Reid, K., et al., Research Needs in Mechanical Systems, Mechanical Engineering 106, $28-43$ (1984). 